\title{
Gelatin hydrogels with eicosapentaenoic acid can prevent osteoarthritis progression in vivo in a mouse model
}

\section{Masanori Tsubosaka}

Kobe University Graduate School of Medicine

\section{Shinsuke Kihara}

Kobe University Graduate School of Medicine

Shinya Hayashi ( $\sim$ s11793290@yahoo.co.jp )

Junpei Nagata

Laboratory of Biomaterials, Institute for Frontier Life and Medical Sciences, Kyoto University

Toshie Kuwahara

Laboratory of Biomaterials, Institute for Frontier Life and Medical Sciences, Kyoto University

\section{Masahiro Fujita}

Kobe University Graduate School of Medicine

\section{Kenichi Kikuchi}

Kobe University Graduate School of Medicine

Yoshinori Takashima

Kobe University Graduate School of Medicine

Tomoyuki Kamenaga

Kobe University Graduate School of Medicine

\section{Yuichi Kuroda}

Kobe University Graduate School of Medicine

\section{Kazuhiro Takeuchi}

Kobe University Graduate School of Medicine

\section{Koji Takayama}

Kobe University Graduate School of Medicine

\section{Shingo Hashimoto}

Kobe University Graduate School of Medicine

\section{Tomoyuki Matsumoto}

Kobe University Graduate School of Medicine

Takahiro Niikura

Kobe University Graduate School of Medicine

Yasuhiko Tabata

Laboratory of Biomaterials, Institute for Frontier Life and Medical Sciences, Kyoto University 


\section{Ryosuke Kuroda}

Kobe University Graduate School of Medicine

\section{Research article}

Keywords: osteoarthritis, eicosapentaenoic acid, omega-3 polyunsaturated fatty acid, gelatin hydrogel

Posted Date: January 28th, 2020

DOI: https://doi.org/10.21203/rs.2.21956/v1

License: (c) (1) This work is licensed under a Creative Commons Attribution 4.0 International License. Read Full License 


\section{Abstract}

\section{Background}

Eicosapentanoic acid (EPA) is an antioxidant and omega-3 polyunsaturated fatty acid that reduces inflammatory cytokine production. Gelatin hydrogel is a release technology that contains a physiologically active substance and releases the substance gradually for an average of $\sim 3$ weeks. Therefore, this study aimed to investigate the effect of EPA-incorporating gelatin hydrogels on osteoarthritis $(\mathrm{OA})$ progression in vivo .

Methods

Ten-week-old male C57BL/6J mice were randomly divided into six groups (six mice each): sham, destabilization of the medial meniscus (DMM), Corn (DMM $+2 \mu \mathrm{l}$ corn oil), EPA-I (DMM $+2 \mu \mathrm{l}$ corn oil +3 $\mathrm{mg} / \mathrm{ml}$ EPA), Control (DMM + control gelatin hydrogels), and EPA-G (DMM $+3 \mathrm{mg} / \mathrm{ml}$ EPA-incorporating gelatin hydrogels) groups. Mice were then euthanized 8 weeks after DMM or sham surgery, and subjected to histological evaluation. Furthermore, MMP-3, MMP-13, IL-1 $\beta$, p-IKK a/ $\beta$, CD86, and CD163 was detected by immunohistochemical staining. F4/80 expression was also assessed using the F4/80 score of synovitis.

Results

Histologic score was significantly improved in EPA-G compared to in EPA-I. MMP-3-, MMP-13-, IL-1 $\beta$-, and p-IKK a/ $\beta$-positive cell ratio was significantly improved in EPA-G compared to in EPA-I. However, CD86and CD163-positive cell ratio was not significantly different between EPA-I and EPA-G. The average-sum F4/80 score of synovitis in EPA-G was significantly improved compared to in EPA-I.

\section{Conclusions}

EPA-incorporating gelatin hydrogels can prevent OA progression in vivo more effectively than single injection of EPA. Our results suggested that intra-articular administration of controlled-release EPA can be a new therapeutic approach for treating $O A$.

\section{Introduction}

Eicosapentaenoic acid (EPA) is an antioxidant and a type of $n-3$ polyunsaturated fatty acid (PUFA) contained in fish oil [1]. Currently, n-3 PUFAs are well-known for their anti-inflammatory and immunomodulatory properties, and for their efficacy in reducing production of inflammatory cytokines [2]. n-3 PUFAs exert their anti-inflammatory effects through multiple mechanisms, including inhibition of arachidonic acid conversion to pro-inflammatory eicosanoids, synthesis of anti-inflammatory products, and downregulation of pro-inflammatory gene expression $[3,4]$. In recent years, the effects of n-3 PUFAs have been extensively studied in cancer, inflammatory bowel disease, and other autoimmune diseases such as and rheumatoid arthritis $[5,6]$. Regarding OA, we previously reported that EPA treatment inhibits 
chondrocyte apoptosis and MMP expression induced by oxidative stress in vitro, and prevents OA progression in vivo [7]. However, in that previous study, weekly injection of EPA into the knee joint was required.

Gelatin hydrogels were developed as a potentially safe drug delivery system that contains physiologically active substance and releases the substance gradually for an average of less than 3 weeks [8]. It was reported that the incorporation of a gelatin hydrogel sheet with growth factors and its subsequent adsorption using electrostatic force preserved the biological activity of the growth factors. Therefore, the preserved bioactive growth factors can be sustainably released until the gelatin hydrogel sheet is degraded. Thus, a gelatin hydrogel sheet can be effectively used to deliver mediations to the applied area owing to its excellent structural and physiological properties while also providing mechanical support to the applied area [9-11]. The effects of gelatin hydrogels are also proved in the field of orthopedic surgery. Oka et al. reported that simvastatin-conjugated gelatin hydrogels promote tendon-bone healing by affecting both angiogenesis and osteogenesis [12]. Another study reported that a combination of BMP-2releasing gelatin/ $\beta$-TCP sponge is a promising technique to induce bone regeneration in bone marrow for treating segmental bone defects induced by $X$-ray irradiation [13]. These findings on the antioxidant action of EPA and the applicability effect of gelatin hydrogels as an innovative drug delivery system suggested that gelatin hydrogels containing EPA might have be more effective than EPA alone in preventing OA progression. We hypothesized that the in vivo effect of gelatin hydrogels containing EPA would be more potent than that of single EPA injection. Therefore, this study aimed to investigate the effect of gelatin hydrogels containing EPA on OA progression in vivo.

\section{Materials And Methods}

This study was performed in strict accordance with the recommendations from the Guide for the Care and Use of Laboratory Animals of the National Institutes of Health (Bethesda, MD, USA). All procedures were approved by the Animal Studies Committee of our institute (permit number: P130108).

\section{Gelatin hydrogel incorporating EPA}

EPA was provided by Sigma-Aldrich (Saint Louis, MO, USA). Gelatin hydrogels containing EPA were produced according to a previous report [14]. Briefly, in the first step, L-lactic acid oligomers (LAo) with a mean molecular weight of 1,000 were synthesized from L-lactide monomers through ring-opening polymerization with stannous octoate as a catalyst and 1-dodecanol as an initiator. LAo-grafted gelatin was synthesized by activating the hydroxyl groups of LAo and mixing them with gelatin solution. EPA/ethanol solution was added to the Lao-grafted gelatin aqueous solution. The reaction mixture was freeze-dried to obtain EPA water-solubilized by LAo-grafted gelatin micelles (EPA-micelles). EPA-micelle aqueous solution and gelatin hydrogel aqueous solution were mixed and adjusted to prepare the desired amount of EPA. Next, 2-ml drops of the mixed solution were placed on a parafilm. Pellets of gelatin hydrogels containing EPA-micelle were prepared through dehydrothermal crosslinking $\left(140^{\circ} \mathrm{C}, 24 \mathrm{~h}\right)$ of gelatin and ethylene oxide gas sterilization. The obtained solid small pellets of gelatin hydrogels 
containing EPA-micelles were used in this study. The small pellets shape hemispherical and have a diameter of about $1.5 \mathrm{~mm}$. EPA content of the pellets was $125 \mu \mathrm{g} / \mu \mathrm{L}$. The gelatin hydrogels were designed to biodegrade over a period of $\sim 3$ weeks under in vivo conditions [15].

\section{Release test of EPA from gelatin hydrogel incorporating EPA}

To investigate the sustained release of EPA in vitro, gelatin hydrogels incorporating EPA were placed in $1.0 \mathrm{ml}$ phosphate-buffered saline (PBS) with $5.0 \mathrm{mg} / \mathrm{ml}$ collagenase. The release test was performed at $37^{\circ} \mathrm{C}$ and the PBS was exchanged at each time-point until gelatin hydrogel was completely dissolved. The supernatant was collected and frozen at $-40{ }^{\circ} \mathrm{C}$ until measurement. The amount of EPA in the supernatant was measured according to previous report using high performance liquid chromatography (HPLC) [16]. The mass of EPA obtained by HPLC measurement at each time-point was measured, and the ratio of the mass compared to that at time-point when gelatin hydrogel was completely dissolved was shown as a graph. This assay repeated 5 times. The sustained release of EPA in vivo, ten-week-old male C57BL/6J mice $(n=35)$ were obtained from the Jackson Laboratory. Gelatin hydrogels were placed into the knee joint cavity. These mice were sacrificed and the gelatin hydrogels were taken from the knee joint cavity at $1,3,7,14,21$, and 28 days after surgery. EPA concentration of gelatin hydrogels were measured by same procedure as in vitro.

\section{In vivo studies}

Ten-week-old male C57BL/6J mice $(\mathrm{n}=72)$ were obtained from the Jackson Laboratory (Bar Harbor, ME) in this experiment. Animals were bred in a mouse house with automatically controlled lightening ( $12 \mathrm{~h}$ on, $12 \mathrm{~h}$ off), and a stable temperature of $23^{\circ} \mathrm{C}$ was maintained throughout the study. Animals also received food and water ad libitum. The mice were anesthetized by intraperitoneal injection of medetomidine $(0.3 \mathrm{mg} / \mathrm{kg})$, midazolam ( $4 \mathrm{mg} / \mathrm{kg})$, and butorphanol $(5 \mathrm{mg} / \mathrm{kg})$, and destabilization of the medial meniscus (DMM) was performed on the right knee joint through transection of the anterior attachment of the medial meniscotibial ligament, as described previously $[17,18]$. The control group was subjected to sham surgery, in which skin and capsule incision and suture were performed on right-knee joints where the ligaments were intact. We randomly divided 72 mice into 6 groups: sham, DMM, Corn (DMM and injection of corn oil), EPA-I (DMM and injection of corn oil and EPA), Control (DMM and control gelatin hydrogels), and EPA-G (DMM and gelatin hydrogels containing EPA). Corn oil $(2 \mu \mathrm{L})$ alone or corn oil (2 $\mu \mathrm{L})$ plus EPA ( $3 \mathrm{mg} / \mathrm{ml})$ was injected once into the knee joint immediately after DMM surgery. Control gelatin hydrogels $(2 \mu \mathrm{L})$ or gelatin hydrogels $(2 \mu \mathrm{L})$ containing EPA $(3 \mathrm{mg} / \mathrm{ml})$ were placed into the knee joint cavity during DMM surgery. The amount of EPA was determined according to a previously reported method [7]. All mice were sacrificed and analyzed at 1 and 8 weeks after DMM or sham surgery, and subjected to histological evaluation. Six mice were analyzed in each group.

\section{Histological evaluation of cartilage degeneration and synovitis}

Mouse knee joints were fixed with $4 \%$ paraformaldehyde for $24 \mathrm{~h}$, decalcified with $14 \%$ EDTA for 7 days, embedded in paraffin. Coronal histological sections were obtained through the joint at $80 \mu \mathrm{m}$ intervals. 
Cartilage destruction was evaluated by Safranin-O and Fast Green staining. Histological evaluation of OA was performed using the cartilage OA-histopathology scoring system of the Osteoarthritis Research Society International (OARSI) [19]. Histological scores were measured in four quadrants (medial femoral condyle, medial tibial plateau, lateral femoral condyle, and lateral tibial plateau) of knee joints at all sectioned levels (eight sections per knee) to obtain summed OA scores. Synovitis was also evaluated using an established scoring system by Lewis et al. and hematoxylin-eosin staining [20]. The average score for changes in synovial lining thickness and cellular density in the synovial stroma (maximum site score 6) of each compartment in the coronal slice was obtained as synovitis score.

\section{Immunohistochemistry}

Deparaffinized sections were digested with proteinase (Dako, Glostrup, Denmark) for 10 min and treated with $3 \%$ hydrogen peroxide (Wako Pure Chemical Industries, Osaka, Japan) to block endogenous peroxidase activity. We assessed F4/80 expression using a previously reported scoring system for immunohistochemistry analysis of immune and inflammatory cell marker [21]. We evaluated CD86 as an M1 macrophage marker and CD163 as an M2 macrophage marker. The sections were treated with a 1:50 dilution of anti-F4/80 (AbD Serotec, Kidlington, UK), anti-CD86 (Bioss Antibodies, Woburn, MA, USA), antiCD163 (Bioss Antibodies), anti-IL 1 $\beta$ (Abcam, Cambridge, UK), phosphor-lkB kinase complex (p-IKK) a/b (Cell Signaling Technology, Danvers, MA, USA), anti-MMP-3 (Santa Cruz Biotechnology, Dallas, TX, USA), or anti-MMP-13 (Abcam) antibody at $4{ }^{\circ} \mathrm{C}$ for $12 \mathrm{~h}$, and subsequently treated with peroxidase-labeled antirabbit or rat immunoglobulin (Histofine Simple Stain MAX PO; Nichirei Bioscience, Tokyo, Japan) at $25^{\circ} \mathrm{C}$ for $30 \mathrm{~min}$. The signal was developed as a brown reaction-product by using peroxidase substrate 3,3'diaminobenzidine (Histofine Simple Stain DAB solution; Nichirei Bioscience). Hematoxylin was used as a counterstain, and then the sections were examined microscopically. One section at the center of the most severe OA lesion in coronal slice was scored. Number of stained cells was counted in three regions, which was randomly defined by one blinded observer, with high magnification fields (40x) in both the superficial and deep zones of cartilage tissue by triple-blinded observers. The range of number of counted cells were about from 60 to 100 in each region. The average percentage of IL-1 $\beta-$, MMP-3-, MMP-13-, p-IKKa/ $\beta-$, CD86-, and CD163-positive cells per total cells was calculated. We also assessed $F 4 / 80$ expression using F4/80 score $[22,23]$. F4/80 positive cells were scored based on numbers of cells as follows: $0=$ no staining, $1=$ minimal to few faintly positive cells, $2=$ scattered single positive cells, $3=$ clusters of two or more positive cells, 4 = larger clusters of positive cells, multifocal to coalescing. The scores were indicated as the total of four different areas of the synovial membrane (superficial layer and deep layer of medial or lateral region in coronal slice).

\section{Cell Culture}

Normal human articular chondrocytes isolated from the knee (NHAC-kn; Lonza Group Ltd., Walkersville, MD, USA) were cultured in $10 \mathrm{ml}$ of Dulbecco's modified Eagle's medium (DMEM) (Gibco; Thermo Fisher Scientific, Inc., Waltham, MA, USA) supplemented with $10 \%$ fetal bovine serum (BioWhittaker; Lonza Group Ltd., Basel, Switzerland) and 100 units $/ \mathrm{ml}$ of penicillin-streptomycin at $37^{\circ} \mathrm{C}$ in a $5 \% \mathrm{CO}_{2}$ atmosphere. Cells at passages 3-5 were used in the experiments. 


\section{Western blotting analysis}

First, $3.0 \times 10^{5}$ chondrocytes were seeded on 6 -well plates and cultured at $37^{\circ} \mathrm{C}$ for $24 \mathrm{~h}$. Next, the medium was replaced with fresh medium, and the cells were incubated for another $24 \mathrm{~h}$. After that, the chondrocytes were treated with or without $10 \mathrm{ng} / \mathrm{ml}$ recombinant human IL-1 (R\&D Systems, McKinnley, MN, USA) for $5,10,15,30$, and 60 min. Stimulation time of IL-1 $\beta$ was determined with reference to previous report [24]. The chondrocytes were washed with Tris-buffered saline with Tween-20 (TBST) and then lysed in a buffer containing $25 \mathrm{mM}$ Tris, $1 \%$ Nonidet P-40, $150 \mathrm{mM} \mathrm{NaCl}, 1.5 \mathrm{mM}$ EGTA, and a protease/phosphatase inhibitor mix (Roche Diagnostics, Basel, Switzerland). Lysates were centrifuged at $4{ }^{\circ} \mathrm{C}$ at $15,000 \times \mathrm{g}$ for $10 \mathrm{~min}$ to remove cellular debris. Next, the lysates with cellular debris removed were collected and mixed with $4 \times$ electrophoresis sample buffer. $15 \mu$ of cell lysates at $1.0 \times 10^{7}$ cells per ml were electrophoresed on a 7.5-15\% SDS-polyacrylamide gradient gel (Biocraft, Tokyo, Japan), and electrically transferred onto a polyvinylidene difluoride blotting membrane (GE Healthcare Life Sciences, Little Chalfont, UK). The membrane was blocked with $5 \%$ skimmed milk in TBST at $25^{\circ} \mathrm{C}$ for $30 \mathrm{~min}$, incubated with antibodies against anti-p-IKKa/ $\beta$ (Cell Signaling Technology) at $4{ }^{\circ} \mathrm{C}$ for $12 \mathrm{~h}$, and incubated with horseradish peroxidase-conjugated goat anti-rabbit immunoglobulin $\mathrm{G}$ secondary antibody at $25^{\circ} \mathrm{C}$ for $30 \mathrm{~min}$. Proteins were subsequently visualized using ECL Plus reagent (GE Healthcare Life Sciences) in a chemilumino analyzer (LAS-3000 mini; Fujifilm, Tokyo, Japan).

Second, $3.0 \times 10^{5}$ chondrocytes were seeded on 6-well plates and cultured at $37^{\circ} \mathrm{C}$ for another $24 \mathrm{~h}$. Next, the medium was replaced with fresh medium, and the cells were incubated for another $24 \mathrm{~h}$. The chondrocytes were then cultured in three settings: under treatment with $10 \mathrm{ng} / \mathrm{ml}$ recombinant human IL$1 \beta$ for the duration that showed the highest $\mathrm{p}-\mathrm{IKKa} / \beta$ level in the above procedure; under the same stimulation for the same duration after pretreatment with $30 \mu \mathrm{g} / \mathrm{ml}$ EPA (Sigma-Aldrich) for $30 \mathrm{~min}$; and without stimulation. The chondrocytes were subsequently subjected to the same procedure as described above.

Finally, $3.0 \times 10^{5}$ chondrocytes were seeded on 6-well plates and cultured at $37^{\circ} \mathrm{C}$ for $24 \mathrm{~h}$. The medium was replaced with fresh medium, and the cells were incubated for another $24 \mathrm{~h}$. After that, the chondrocytes were subjected to five different treatments: treatment with $10 \mathrm{ng} / \mathrm{ml}$ recombinant human IL$1 \beta$ for $24 \mathrm{~h}$; the same treatment for $24 \mathrm{~h}$ after pretreatment with EPA (Sigma-Aldrich) at 10 (low dose), 30 (medium dose), or $50 \mathrm{\mu g} / \mathrm{ml}$ (high dose) for $30 \mathrm{~min}$; or without treatment. Next, the chondrocytes were subjected to the same procedure as described above, except that membranes were incubated with antibodies against anti-MMP-13 instead of antibodies against anti-p-IKKa/ $\beta$. The expression of alphatubulin protein was detected using rabbit anti-alpha-tubulin polyclonal antibody (Abcam) as a primary antibody. Protein expression was determined through semi-quantification of proteins in digitally captured images using the National Institutes of Health ImageJ software (National Institutes of Health, Maryland, USA, http://imagej.nih.gov/ij/). The number of each sample was 5.

\section{Statistical analysis}


Statistical analysis was performed using one-way or two-way analysis of variance with Tukey's post-hoc test for multiple comparisons of paired samples. The Mann-Whitney $\mathrm{U}$ test was used to compare between two groups. Results are presented as mean values with standard deviation (S.D). Differences in mean values were considered statistically significant at $p<0.05$.

\section{Results}

\section{Release and degradation EPA from gelatin hydrogels containing statin micelles}

Figure $1 \mathrm{~A}$ shows the time profiles of EPA release from gelatin hydrogels containing EPA micelles in PBS with collagenase. In the presence of collagenase, EPA was released with time from the gelatin hydrogels. As shown in Fig. 1A, the gelatin hydrogels were biodegraded over a period of $\sim 3$ weeks in vivo, in accordance with their design. Figure 1B shows the time profiles of EPA release from gelatin hydrogels containing EPA micelles in the joints of mice. According to Fig. 1B, the gelatin hydrogels were also biodegraded over a period of $\sim 4$ weeks in vivo.

\section{EPA prevented OA progression in mice}

The average sum of cartilage OA histopathology scores at 1 week in the sham, DMM, Corn, EPA-I, Control, and EPA-G groups were $0.42 \pm 0.19,1.3 \pm 0.19,1.2 \pm 0.27,0.67 \pm 0.12,1.3 \pm 0.22$, and $0.75 \pm 0.14$, respectively (Fig. 2I), with no significant difference between the EPA-I and EPA-G groups (Fig. 2B, D). The average sum scores at 8 weeks in the sham, DMM, Corn, EPA-I, Control, and EPA-G groups were $0.96 \pm$ $0.17,3.2 \pm 0.37,2.8 \pm 0.22,2.4 \pm 0.35,2.9 \pm 0.19$, and $1.2 \pm 0.27$, respectively (Fig. $2 \mathrm{~J}$ ), with significant difference EPA-I and EPA-G groups (Fig. $2 F, H$ ), with no significant difference between the sham and EPAG groups (Fig. 2E, H). No serious adverse event was observed throughout this study.

\section{EPA prevented severe synovitis during OA progression}

The average sum of synovitis severity scores at 1 week after surgery in the sham, DMM, Corn, EPA-I, Control, and EPA-G groups were $0.83 \pm 0.37,5.7 \pm 0.47,5.0 \pm 0.58,2.3 \pm 0.47,5.3 \pm 0.47$, and $2.2 \pm 0.37$, respectively (Fig. 3I), with no significant difference between the EPA-I and EPA-G groups (Fig. 3B, D). The average-sum scores at 8 weeks after surgery in the sham, DMM, Corn, EPA-I, Control, and EPA-G groups were $1.5 \pm 0.5,4.0 \pm 0.58,3.8 \pm 0.69,3.8 \pm 0.69,4.0 \pm 0.58$, and $1.8 \pm 0.69$, respectively (Fig. $3 \mathrm{~N}$ ), with significant difference between the EPA-I and EPA-G groups (Fig. 3F, H), with no significant difference between the sham and EPA-G groups (Fig. 3E, H).

\section{EPA prevented macrophage infiltration in synovial tissues}

Results of immunohistochemistry analysis showed that the average sum of F4/80 synovitis scores at 1 week after surgery in the sham, DMM, Corn, EPA-I, Control, and EPA-G groups were $0.83 \pm 0.69,10.2 \pm 1.3$, 
$8.2 \pm 0.69,3.3 \pm 0.47,9.2 \pm 0.69$, and $2.8 \pm 0.69$, respectively (Fig. 4l), with no significant difference between the EPA-I and EPA-G groups (Fig. 4B, D). The average sum scores at 8 weeks after surgery in the sham, DMM, Corn, EPA-I, Control, and EPA-G groups were $1.3 \pm 0.47,7.3 \pm 0.94,5.7 \pm 0.47,5.2 \pm 0.69,6.5 \pm$ 0.96 , and $2.7 \pm 0.74$, respectively (Fig. $4 \mathrm{~J}$ ), with significant difference between the EPA-I and EPA-G groups (Fig. 4F, H).

There was no significant difference in the ratio of CD86-positive cells at 1 week after surgery between the EPA-I and EPA-G groups (Fig. 5B, D). In addition, the ratio of CD86-positive cells at 8 weeks after surgery showed no significant difference in the EPA-I and EPA-G groups (Fig. 5F, H). Moreover, the ratio of CD163positive cells at 1 week after surgery showed no significant difference in the EPA-I and EPA-G groups (Fig. 6B, D). Furthermore, the ratio of CD163-positive cells at 8 weeks after surgery showed no significant difference in the EPA-I and EPA-G groups (Fig. 6F, H).

\section{EPA prevented OA progression by suppressing IL-1 $\beta$, p- $\mathrm{IKKa} / \beta$, and MMP levels}

The results of immunohistochemical analysis showed that the ratio of IL-1 $\beta-$, p-IKKa/ $\beta-$, MMP-3-, and MMP-13-positive cells at 1 week after surgery showed no significant difference between the EPA-I and EPA-G groups (Supplemental Fig. S1B, D, F, H, J, L; Fig. S2B, D). The ratios of IL-1 $\beta-$, p-IKKa/ $\beta-$, MMP-3-, and MMP-13-positive cells at 8 weeks after surgery showed significant difference in the EPA-I and EPA-G groups (Supplemental Fig. S1B, D, F, H, J, L; Fig. S2F, H).

Mice were obtained from the Jackson Laboratory, and all obtained 72 mice were used in this experiment. There was no adverse event after sham or DMM surgery. Primary antibody negative controls for immunohistochemical analysis were shown in Fig. S3.

\section{Enhanced expression of inflammatory transcription factors in response to IL1 $\beta$ stimulation in vitro}

To investigate the role of EPA in signal transduction, phosphorylation of IKKa/ $\beta$ in NHAC-kn treated with $10 \mathrm{ng} / \mathrm{ml} \mathrm{IL1} \beta$ was assessed. The results confirmed timedependent IL1 $\beta$-induced phosphorylation of IKKa/ $\beta$. Particularly, $p-I K K a / \beta$ level markedly increased at 15 min after treatment (Fig. 8A). Therefore, $\mathrm{NHAC}-\mathrm{kn}$ was treated with IL1 $\beta$ for $15 \mathrm{~min}$ in the subsequent experiment. Western blotting results showed that the level of phosphorylated IKKa/b, which is a kinase necessary for the activation of nuclear factor NF-KB, was significantly higher following treatment without EPA than following treatment with EPA (Fig. 8B). Moreover, MMP-13 level following treatment without EPA was significantly higher than that following treatment with EPA. Furthermore, the effect was dose-dependent; the higher the dose, the more remarkable the effect (Fig. 8C). These results indicated that EPA suppressed IL-1 $\beta$-induced phosphorylation of $\mathrm{IKKa} / \mathrm{b}$, and thus prevent the activation of the NF-KB signaling pathway. 


\section{Discussion}

The most important finding in this study was that the in vivo effect of gelatin hydrogels containing EPA was more potent than that of a single EPA injection, which supported our study hypothesis.

In the current study, immunohistochemical analysis showed that the ratio of IL-1 $\beta$-positive cells at 1 week after surgery was significantly reduced in the EPA-I and EPA-G groups. Furthermore, IL-1 $\beta$-induced increases in $\mathrm{p}-\mathrm{IKKa} / \mathrm{b}$ and MMP-13 levels were significantly suppressed by treatment with EPA, compared with those after treatment without EPA at 1 week after surgery. However, there was no significant difference in macrophage infiltration and expression of IL1- $\beta, p-I K K$, and MMPs between the group treated with corn oil and single EPA injection at 8 weeks after surgery, although there were significant differences between the group treated with control gelatin hydrogels and EPA-incorporating hydrogels. Here, IL-1 $\beta$ expression increased with OA progression, and increased IL-1 $\beta$ expression causes the activation of the NF-KB pathway and expression of MMP-3 and -13 , ultimately causing matrix degradation of articular cartilage. Taken together, EPA suppressed IL-1 $\beta$ expression, thus inhibiting the activation of the downstream pathways of IL-1 $\beta$ and ultimately preventing matrix loss. However, the inflammation inhibitory effect of single EPA injection was diminished at 8 weeks after surgery. In contrast, the effect of EPA-incorporating hydrogels was prolonged up to 8 weeks after surgery. Although we previously reported that EPA treatment prevents OA progression in vivo [7], weekly injection of EPA into the knee joint was required. This weekly injection was thought to be highly invasive in clinical applications, so we examined the effects of single intervention. As a result, there was no significant difference in prevention of OA progression at 8 weeks after DMM surgery between weekly injection of EPA and once placement of gelatin hydrogel incorporating EPA. The MMP-13 level of chondrocytes following treatment with IL-1 $\beta$ under existence of EPA depended on EPA dose. Furthermore, several papers have been reported on the possibility that increasing EPA intake may have systemic beneficial effects $[25,26]$. Therefore, the effect of EPA was considered to be dose-dependent in both local and systemic environment, and it is necessary to carefully examine the optimal amount in case of clinical application.

Matsuzaki et al. reported that gelatin hydrogels incorporating rapamycin gradually release rapamycin within approximately 2 days in vitro, but they release rapamycin for approximately 10 weeks in vivo [14]. Furthermore, gelatin hydrogels incorporating simvastatin have been reported to exhibit sustained release for at least 3 weeks in vitro and 4 weeks in vivo $[15,27]$. The present study involved a formulation of hydrogel incorporating EPA solubilized in water into gelatin micelles. The in vitro sustained release test showed that EPA was released after the decomposition of the gelatin hydrogel. This indicated that the decomposition of gelatin is a rate-limiting step for the release of EPA from hydrogel, allowing EPA to be released slowly over a long period in vivo. Consequently, the EPA-incorporating gelatin hydrogel prevented OA progression in vivo more effectively than a single injection of EPA did.

There are two opposite responses elicited by activated macrophages. Macrophages are known to induce pro- and anti-inflammatory responses that mediate matrix destruction or deposition $[28,29]$. The proinflammatory M1 macrophage produce cytokines, such as tumor necrosis factors, IL-1 and IL-6, 
chemokines monocyte chemoattractant protein-1, and growth factors, such as vascular endothelial growth factor. It also enhances oxidative stress promoters, such as reactive oxygen species and reactive nitrogen species, which in turn activates the NFKB signaling pathway, causing destruction of cartilage matrix [30]. In contrast, the anti-inflammatory M2 macrophage secretes high levels of anti-inflammatory mediators, such as IL-10, which are necessary to regulate inflammation [31]. In the current study, EPA suppressed the number of M1 macrophages at 1-week after surgery, whereas gelatin hydrogels containing EPA suppressed the number of M1 macrophages even at 8 weeks after surgery. However, EPA did not affect the number of M2 macrophages. Thus, EPA was thought to prevent OA progression by suppressing pro-inflammatory action and inhibiting both the IL-1 $\beta$ and NF-KB pathways.

\section{Conclusions}

We investigated the effect of gelatin hydrogels containing EPA on OA progression in vivo. The EPAcontaining gelatin hydrogels could prevent OA progression more effectively than a single injection of EPA in vivo in a DMM mouse model. Our results suggested that intra-articular administration of controlledrelease EPA can be a new therapeutic approach for treating patients with $O A$.

\section{List Of Abbreviations}

Eicosapentaenoic acid (EPA)

polyunsaturated fatty acid (PUFA)

L-lactic acid oligomers (LAo)

EPA water-solubilized by LAo-grafted gelatin micelles (EPA-micelles)

phosphate-buffered saline (PBS)

high performance liquid chromatography (HPLC)

destabilization of the medial meniscus (DMM)

Osteoarthritis Research Society International (OARSI)

phosphor-IkB kinase complex (p-IKK)

normal human articular chondrocytes isolated from the knee (NHAC-kn)

Dulbecco's modified Eagle's medium (DMEM)

Tris-buffered saline with Tween-20 (TBST)

standard deviation (S.D) 


\section{Declarations}

\section{Acknowledgments}

We thank Ms. Kyoko Tanaka, Ms. Minako Nagata, and Ms. Maya Yasuda for their technical assistance.

\section{Author contributions}

All authors have made substantial contributions to (1) the conception and design of the study, or acquisition of data, or analysis and interpretation of data; (2) drafting the article or revising it critically for important intellectual content; and (3) final approval of the version to be submitted. The specific contributions of the authors are as follows:

(1) Conception and design of the study: SHayashi, MT, SK

(2) Analysis and interpretation of the data: MT, SK, JN, YT, TK, YK, KTakeuchi

(3) Drafting of the article: MT, SHayashi, KS

(4) Critical revision of the article for important intellectual content: MT, SH, KTakayama, SHashimoto, TM, TN, YT, RK

(5) Final approval of the article: MT, SK, SHayashi, JN, TKuwahara, MF, KK, YT, TKamenaga, YK, KTakeuchi, KTakayama, SHashimoto, TM, TN, YT, RK

(6) Statistical expertise: MT, SHayashi

(7) Collection and assembly of data: MT, SK, SHayashi

\section{Funding}

None.

\section{Availability of data and materials}

The datasets used and/or analyzed during the current study are available from the corresponding author on reasonable request. 
Not applicable.

\section{Consent for publication}

Not applicable.

\section{Competing interests}

The authors have no competing interests to declare.

\section{References}

[1] Zainal Z, Longman AJ, Hurst S, Duggan K, Caterson B, Hughes CE, et al. Relative efficacies of omega-3 polyunsaturated fatty acids in reducing expression of key proteins in a model system for studying osteoarthritis. Osteoarthritis Cartilage 2009;17:896-905.

[2] Curtis CL, Rees SG, Little CB, Flannery CR, Hughes CE, Wilson C, et al Pathologic indicators of degradation and inflammation in human osteoarthritic cartilage are abrogated by exposure to $n-3$ fatty acids. Arthritis Rheum 2002;46:1544-53.

[3] Oh DY, Talukdar S, Bae EJ, Imamura T, Morinaga H, Fan W, et al. GPR120 is an omega-3 fatty acid receptor mediating potent anti-inflammatory and insulin-sensitizing effects. Cell 2010;142:687-98.

[4] Serhan CN, Petasis NA. Resolvins and protectins in inflammation resolution. Chem Rev 2011;111:5922-43.

[5] Goldberg RJ, Katz J. A meta-analysis of the analgesic effects of omega-3 polyunsaturated fatty acid supplementation for inflammatory joint pain. Pain 2007;129:210-23.

[6] Hurst S, Zainal Z, Caterson B, Hughes CE, Harwood JL. Dietary fatty acids and arthritis. Prostaglandins Leukot Essent Fatty Acids 2010;82:315-8.

[7] Sakata S, Hayashi S, Fujishiro T, Kawakita K, Kanzaki N, Hashimoto S, et al. Oxidative stressinduced apoptosis and matrix loss of chondrocytes is inhibited by eicosapentaenoic acid. J Orthop Res 2015;33:359-65.

[8] Tabata Y, Ikada Y. Vascularization effect of basic fibroblast growth factor released from gelatin hydrogels with different biodegradabilities. Biomaterials 1999;20:2169-75.

[9] Haraguchi T, Okada K, Tabata Y, Maniwa Y, Hayashi Y, Okita Y. Controlled release of basic fibroblast growth factor from gelatin hydrogel sheet improves structural and physiological properties of vein graft in 
rat. Arterioscler Thromb Vasc Biol2007;27:548-55.

[10] Tabata Y, Nagano A, Ikada Y. Biodegradation of hydrogel carrier incorporating fibroblast growth factor. Tissue Eng 1999;5:127-38.

[11] Tambara K, Premaratne GU, Sakaguchi G, Kanemitsu N, Lin X, Nakajima H, et al. Administration of control-released hepatocyte growth factor enhances the efficacy of skeletal myoblast transplantation in rat infarcted hearts by greatly increasing both quantity and quality of the graft. Circulation 2005;112:12934.

[12] Oka S, Matsumoto T, Kubo S, Matsushita T, Sasaki H, Nishizawa Y, et al. Local administration of low-dose simvastatin-conjugated gelatin hydrogel for tendon-bone healing in anterior cruciate ligament reconstruction. Tissue Eng 2013;19:1233-43.

[13] Yamamoto M, Hokugo A, Takahashi Y, Nakano T, Hiraoka M, Tabata Y. Combination of BMP-2releasing gelatin/beta-TCP sponges with autologous bone marrow for bone regeneration of X-rayirradiated rabbit ulnar defects. Biomaterials 2015;56:18-25.

[14] Matsuzaki T, Matsushita T, Tabata Y, Saito T, Matsumoto T, Nagai K, et al. Intra-articular administration of gelatin hydrogels incorporating rapamycin-micelles reduces the development of experimental osteoarthritis in a murine model. Biomaterials 2014;35:9904-11.

[15] Tanigo T, Takaoka R, Tabata Y. Sustained release of water-insoluble simvastatin from biodegradable hydrogel augments bone regeneration. J Control Release 2010;19:201-6.

[16] Uchida Y, Masui T, Nakano K, Yogo A, Sato A, Nagai K, et al. Clinical and experimental studies of intraperitoneal lipolysis and the development of clinically relevant pancreatic fistula after pancreatic surgery. Br J Surg. 2019;106:616-25.

[17] Glasson SS, Blanchet TJ, Morris EA. The surgical destabilization of the medial meniscus (DMM) model of osteoarthritis in the 129/SvEv mouse. Osteoarthritis Cartilage 2007;15:1061-9.

[18] Hashimoto S, Rai MF, Janiszak KL, Cheverud JM, Sandell LJ. Cartilage and bone changes during development of post-traumatic osteoarthritis in selected LGXSM recombinant inbred mice. Osteoarthritis Cartilage 2012;20:562-71.

[19] Glasson SS, Chambers MG, Van Den Berg WB, Little CB. The OARSI histopathology initiative recommendations for histological assessments of osteoarthritis in the mouse. Osteoarthritis Cartilage 2010;18:S17-23.

[20] Lewis JS, Hembree WC, Furman BD, Tippets L, Cattel D, Huebner JL, et al. Acute joint pathology and synovial inflammation is associated with increased intra-articular fracture severity in the mouse knee. Osteoarthritis Cartilage. 2011;19:864-73. 
[21] Fujiwara N, Kobayashi K. Macrophages in inflammation. Curr Drug Targets Inflamm Allergy 2005;4:281-6.

[22] Willis CR, Seamons A, Maxwell J, Treuting PM, Nelson L, Chen G, et al. Interleukin-7 receptor blockade suppresses adaptive and innate inflammatory responses in experimental colitis. J Inflamm (Lond). 2012;9:39

[23] Yamada J, Tsuji K, Miyatake K, Matsukura Y, Abula K, Inoue M, et al. Follistatin alleviates synovitis and articular cartilage degeneration induced by carrageenan. Int J Inflam. 2014;2014:959271.

[24] Kihara S, Hayashi S, Hashimoto S, Kanzaki N, Takayama K, Matsumoto T, et al. Cyclin-Dependent Kinase Inhibitor-1-Deficient Mice Are Susceptible to Osteoarthritis Associated With Enhanced Inflammation. J Bone Miner Res. 2018;33:2242.

[25] Rees D, Miles EA, Banerjee T, Wells SJ, Roynette CE, Wahle KW, et al. Dose-related effects of eicosapentaenoic acid on innate immune function in healthy humans: a comparison of young and older men. Am J Clin Nutr. 2006;83:331-42.

[26] Cazzola R, Russo-Volpe S, Miles EA, Rees D, Banerjee T, Roynette CE, et al. Age- and dosedependent effects of an eicosapentaenoic acid-rich oil on cardiovascular risk factors in healthy male subjects. Atherosclerosis. 2007;193:159-67.

[27] Tanaka T, Matsushita T, Nishida K, Takayama K, Nagai K, Araki D, et al. Attenuation of osteoarthritis progression in mice following intra-articular administration of simvastatin-conjugated gelatin hydrogel. J Tissue Eng Regen Med 2019;13:423-32.

[28] Mantovani A, Sica A, Sozzani S, Allavena P, Vecchi A, Locati M. The chemokine system in diverse forms of macrophage activation and polarization. Trends Immunol 2004;25:677-86.

[29] Murray PJ, Wynn TA. Obstacles and opportunities for understanding macrophage polarization. J Leukoc Biol 2011;89:557-63.

[30] Haddad JJ. Antioxidant and prooxidant mechanisms in the regulation of redox(y)-sensitive transcription factors. Cell Signal 2002;14:879-97.

[31] Martinez FO, Sica A, Mantovani A, Locati M. Macrophage activation and polarization. Front Biosci 2008;13:453-61.

\section{Additional File}

Additional file 1.

Supplementary Fig. $\mathbf{S 1}$ Immunohistochemical analysis showed the average-sum ratio of IL-1 $\beta-$, p-IKKa/ $\beta$-, and MMP-13-positive cells 
Immunohistochemical analysis showed the average-sum ratio of IL-1 $\beta$-, $\mathrm{p}-\mathrm{IKKa} / \beta$-, and MMP-13-positive cells at 1 week after surgery. The ratio of IL-1 $\beta$-positive cells at 1 week in the sham, DMM, Corn, EPA-I, Control, and EPA-G groups were $9.3 \pm 1.5,24.5 \pm 1.4,19.9 \pm 1.8,12.2 \pm 1.4,23.7 \pm 1.9$, and $14.0 \pm 1.7$, respectively. The ratio of $\mathrm{p}-\mathrm{IKKa} / \beta$-positive cells at 1 week in the sham, DMM, Corn, EPA-I, Control, and EPA-G groups were $7.5 \pm 1.5,21.4 \pm 2.4,19.5 \pm 2.4,13.2 \pm 2.0,21.1 \pm 3.2$, and $14.6 \pm 1.5$, respectively. The ratio of MMP-13-positive cells at 1 week in the sham, DMM, Corn, EPA-I, Control, and EPA-G groups were $9.5 \pm 1.0,26.8 \pm 1.1,21.6 \pm 3.4,11.9 \pm 1.9,23.6 \pm 1.8$, and $11.4 \pm 0.94$, respectively. The ratios of IL-1 $\beta-, p-$ IKKa/ $\beta$-, and MMP-13-positive cells at 1 week showed significant difference between the sham and DMM groups, Corn and EPA-I groups, and Control and EPA-G groups, and no significant difference between the EPA-I and EPA-G groups. Immunohistochemical evaluation of IL-1 $\beta, \mathrm{p}-\mathrm{IKKa} / \beta$ and MMP-13 revealed a significant decrease in OA after EPA treatment.

Results are presented as mean values with standard deviation.

DMM: destabilization of the medial meniscus, OA: osteoarthritis, EPA: eicosapentaenoic acid

Supplementary Fig. S2 Immunohistochemical analysis using the average-sum ratio of MMP-3-positive cells

Immunohistochemical analysis showed the average-sum ratio of MMP-3-positive cells at 1 week and 8 weeks after surgery. The ratio of MMP-3-positive cells at 1 week in the sham, DMM, Corn, EPA-I, Control, and EPA-G groups were $8.7 \pm 1.0,19.5 \pm 1.9,17.8 \pm 1.2,11.4 \pm 3.1,19.2 \pm 1.4$, and $12.9 \pm 1.3$, respectively, with significant difference between the sham and DMM groups, Corn and EPA-I groups, and Control and EPA-G groups, no significant difference between the EPA-I and EPA-G groups. The ratio of MMP-3-positive cells at 8 weeks in the sham, DMM, Corn, EPA-I, Control, and EPA-G groups were $14.8 \pm 3.0,38.7 \pm 3.5$, $37.0 \pm 5.1,29.8 \pm 6.2,38.0 \pm 3.8$, and $16.2 \pm 2.5$, respectively, with significant difference between the sham and DMM groups, and Control and EPA-G groups, no significant difference between the Corn and EPA-I groups, and EPA-I and EPA-G groups. Immunohistochemical evaluation of MMP-3 revealed a significant decrease in OA after EPA treatment especially in the group treated with EPA-incorporating gelatin hydrogels at 8 weeks after surgery.

Results are presented as mean values with standard deviation.

DMM: destabilization of the medial meniscus, OA: osteoarthritis, EPA: eicosapentaenoic acid

Supplementary Fig. S3 Primary antibody negative controls for immunohistchemical analysis.

\section{Figures}


Figure $1(\mathrm{~A})$

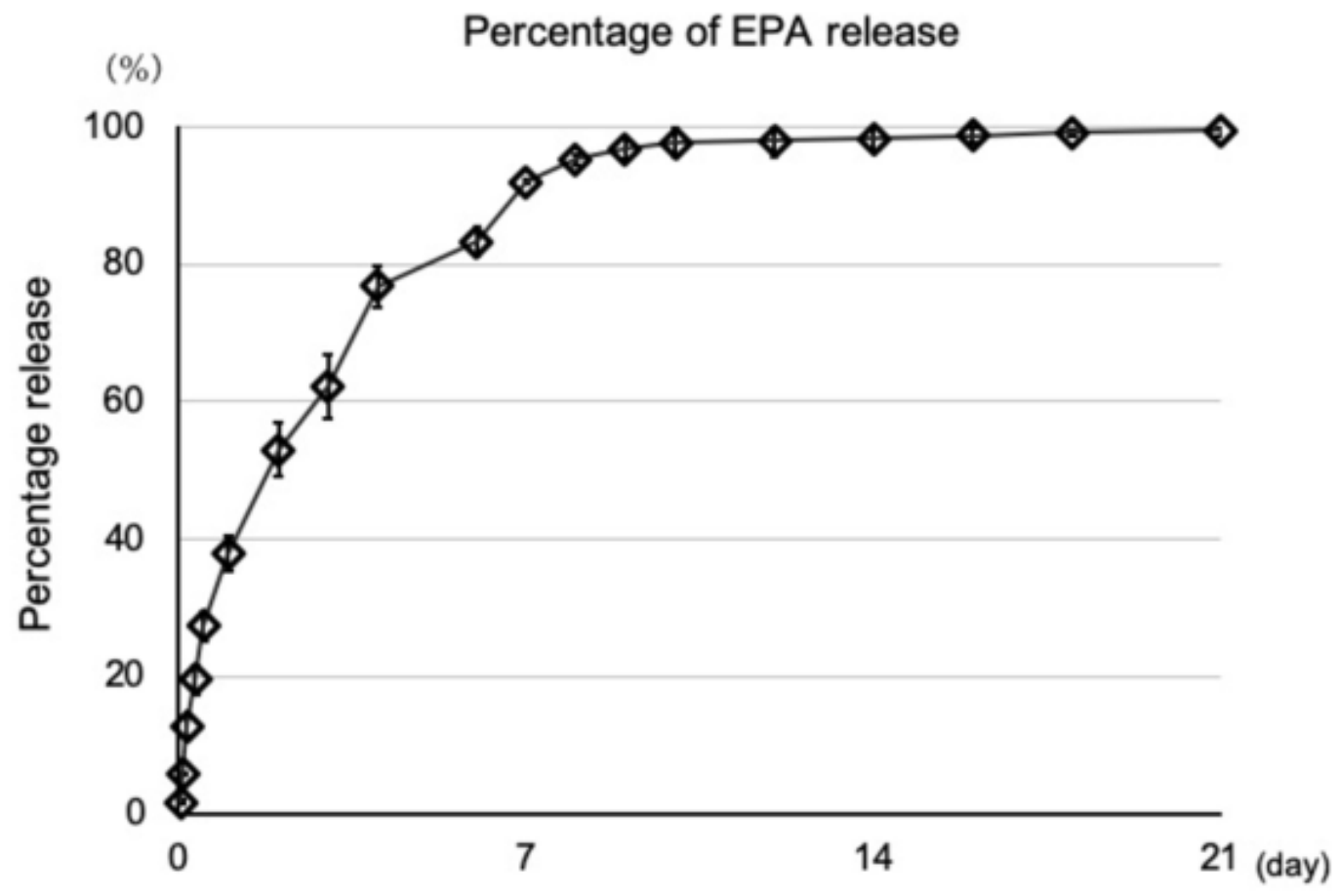

Figure 1(B)

(\%)

Percentage of EPA release

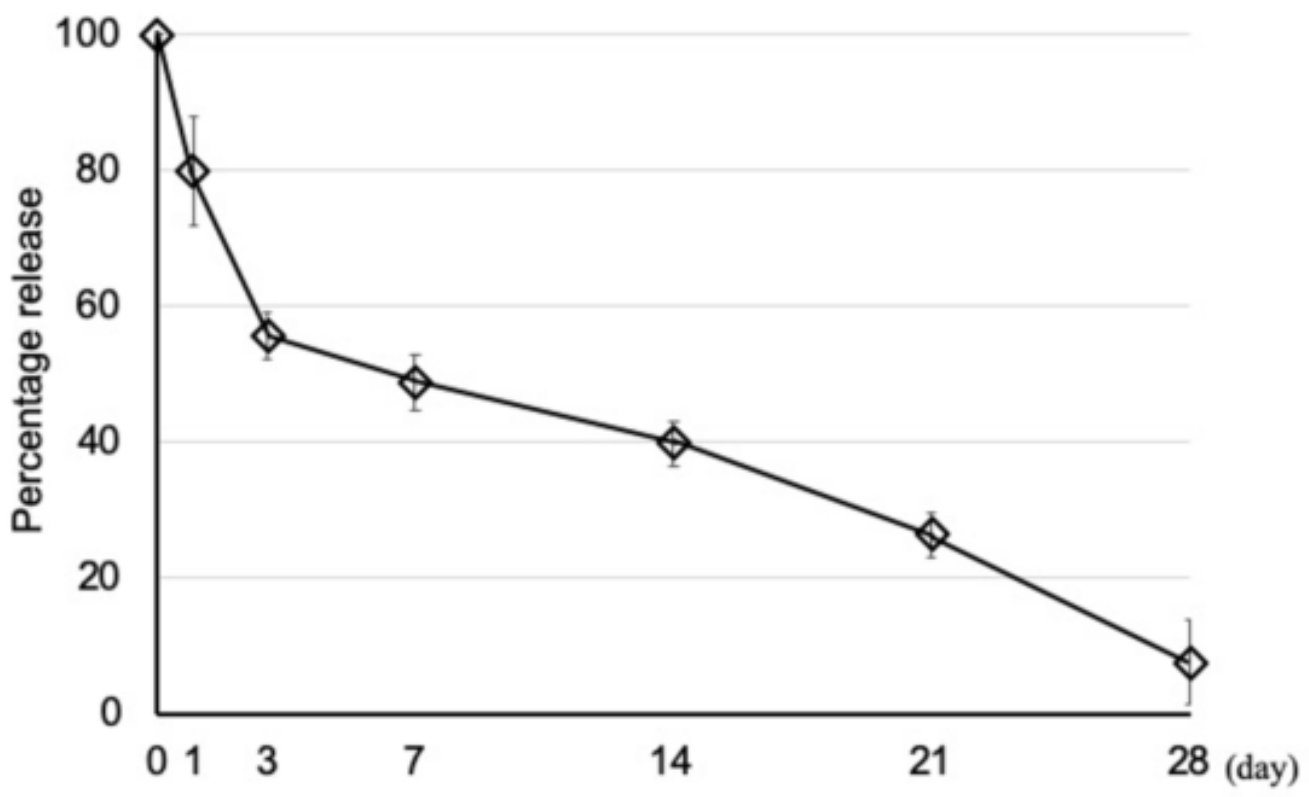

Figure 1

1 Time profiles of eicosapentaenoic acid (EPA) release in vitro and in vivo (A) Time profiles of EPA release from EPA micelle incorporating gelatin hydrogels in PBS containing collagenase. The gelatin hydrogels in this study were designed to biodegrade over a period of $\sim 3$ weeks under in vivo conditions. (B) Time profiles of EPA release from gelatin hydrogels containing EPA micelles in the joints of mice. The gelatin hydrogels were also biodegraded over a period of $\sim 4$ weeks in vivo. 


\section{Figure. 2 Safranin-O staining : Evaluation of Osteoarthritis}

1 week post-DMM 8 weeks post-DMM 1 week

(A)

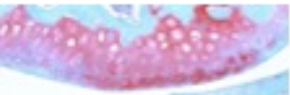

Sham

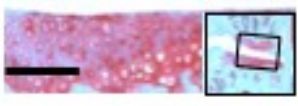

DMM + (B)

(B)

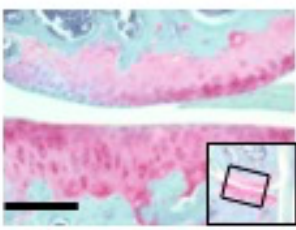

(C)

$\mathrm{DMM}+$ Control-Gel

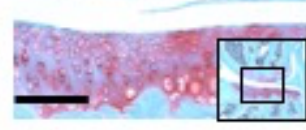

$\mathrm{DMM}+$

(D)

EPA-Gel
(E)

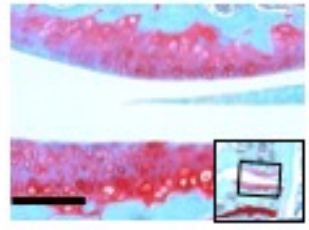

(F)

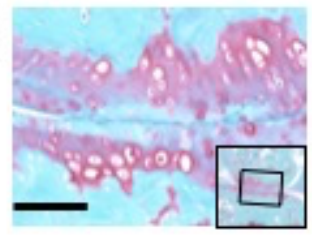

(G)

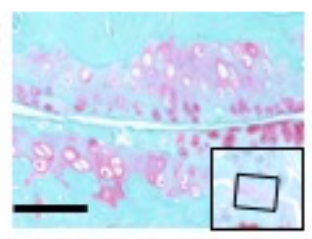

$(\mathrm{H})$

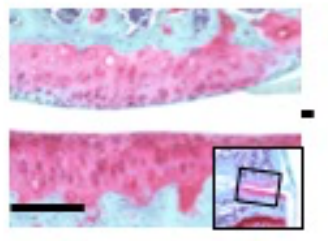

scale bar $=100 \mu \mathrm{m}$
OARSI Histological Score

(I)

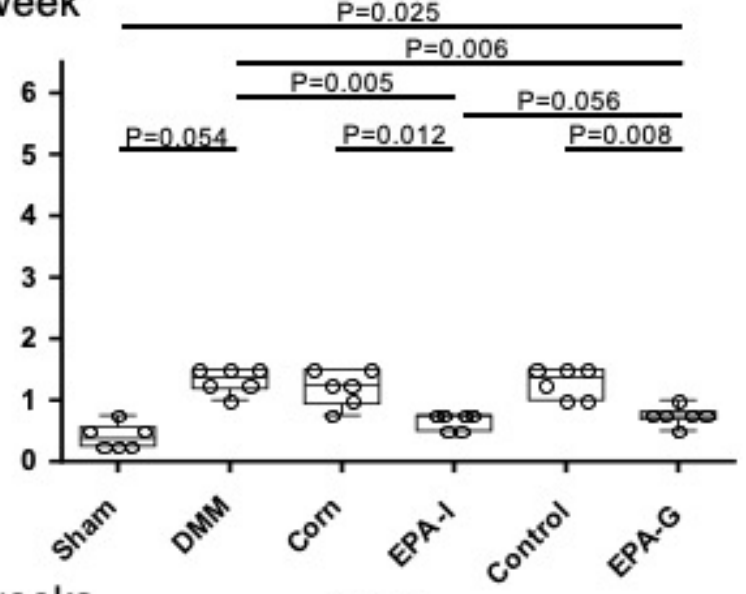

8 weeks

(J)

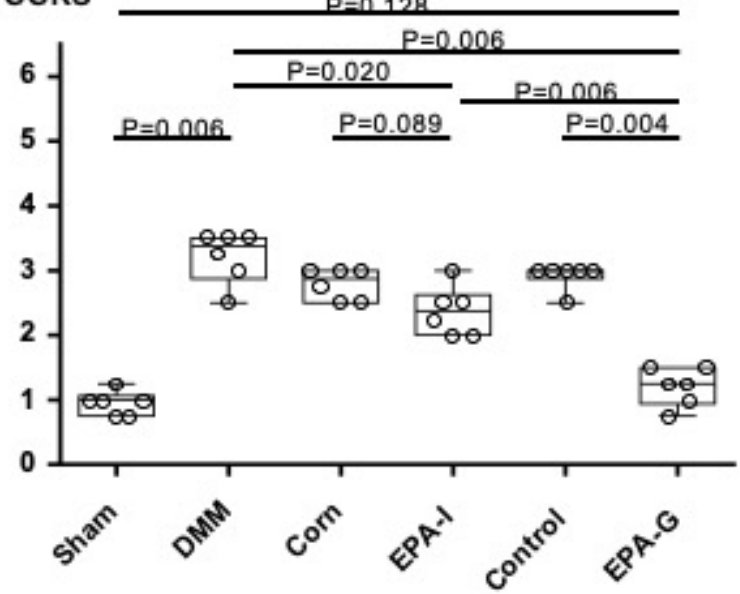

Figure 2

Histologic features for calculation of summed OA scores by the OARSI histological score Histologic features in coronal slice of the knee joints at 1 week and 8 weeks after surgery for calculation of summed OA scores by the OARSI histological score. The average sum scores at 1 week showed significant differences between the sham and DMM groups, Corn and EPA-I groups, and Control and EPA-G groups, and no significant difference between the EPA-I and EPA-G groups. The average sum scores at 8 weeks showed significant difference between the sham and DMM groups, Control and EPA-G groups, and EPA-I and EPA-G groups, and no significant difference between the Corn and EPA-I groups, and sham and EPA-G groups. Histologic evaluation revealed a significant decrease in OA after EPA treatment, especially in the group treated with EPA-incorporating gelatin hydrogels at 8 weeks after surgery. DMM: destabilization of the medial meniscus, OA: osteoarthritis, OARSI: Osteoarthritis Research Society International, EPA: eicosapentaenoic acid 


\section{Figure. 3 Hematoxylin-Eosin staining : Evaluation of Synovitis}

1 week post-DMM 8 weeks post-DMM 1 week

\section{Severity of Synovitis}

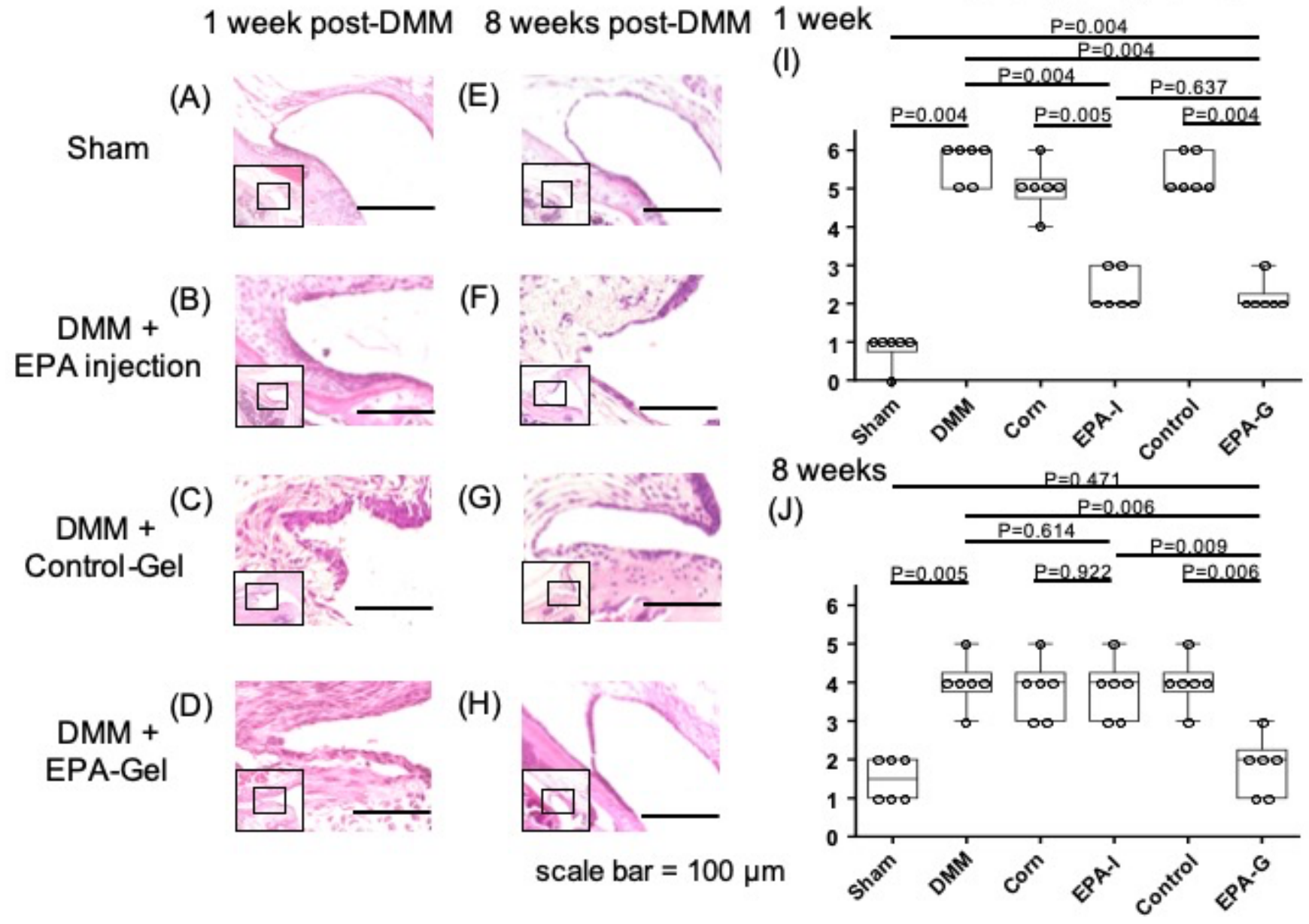

Figure 3

Synovitis evaluations using OARSI-recommended scoring systems Synovitis in hematoxylin and eosinstained sections was evaluated using OARSI-recommended scoring systems. The average score of each compartment in the coronal slice was obtained from synovitis scores at 1 week and 8 weeks after surgery. The average sum scores at 1 week showed significant difference between the sham and DMM groups, Corn and EPA-I groups, and Control and EPA-G groups, and no significant difference between the EPA-I and EPA-G groups. The average sum scores at 8 weeks showed significant difference between the sham and DMM groups, Control and EPA-G groups, and EPA-I and EPA-G groups, and no significant difference between the Corn and EPA-I groups, and sham and EPA-G groups. Synovitis evaluation revealed a significant decrease in OA after EPA treatment, especially in the group treated with EPAincorporating gelatin hydrogels at 8 weeks after surgery. DMM: destabilization of the medial meniscus, OARSI: Osteoarthritis Research Society International OA: osteoarthritis, EPA: eicosapentaenoic acid 


\section{Figure. 4 Immunohistochemical analysis of $\mathrm{F} 4 / 80$}

1 week post-DMM 8 weeks post-DMM 1 week

F4/80 Score

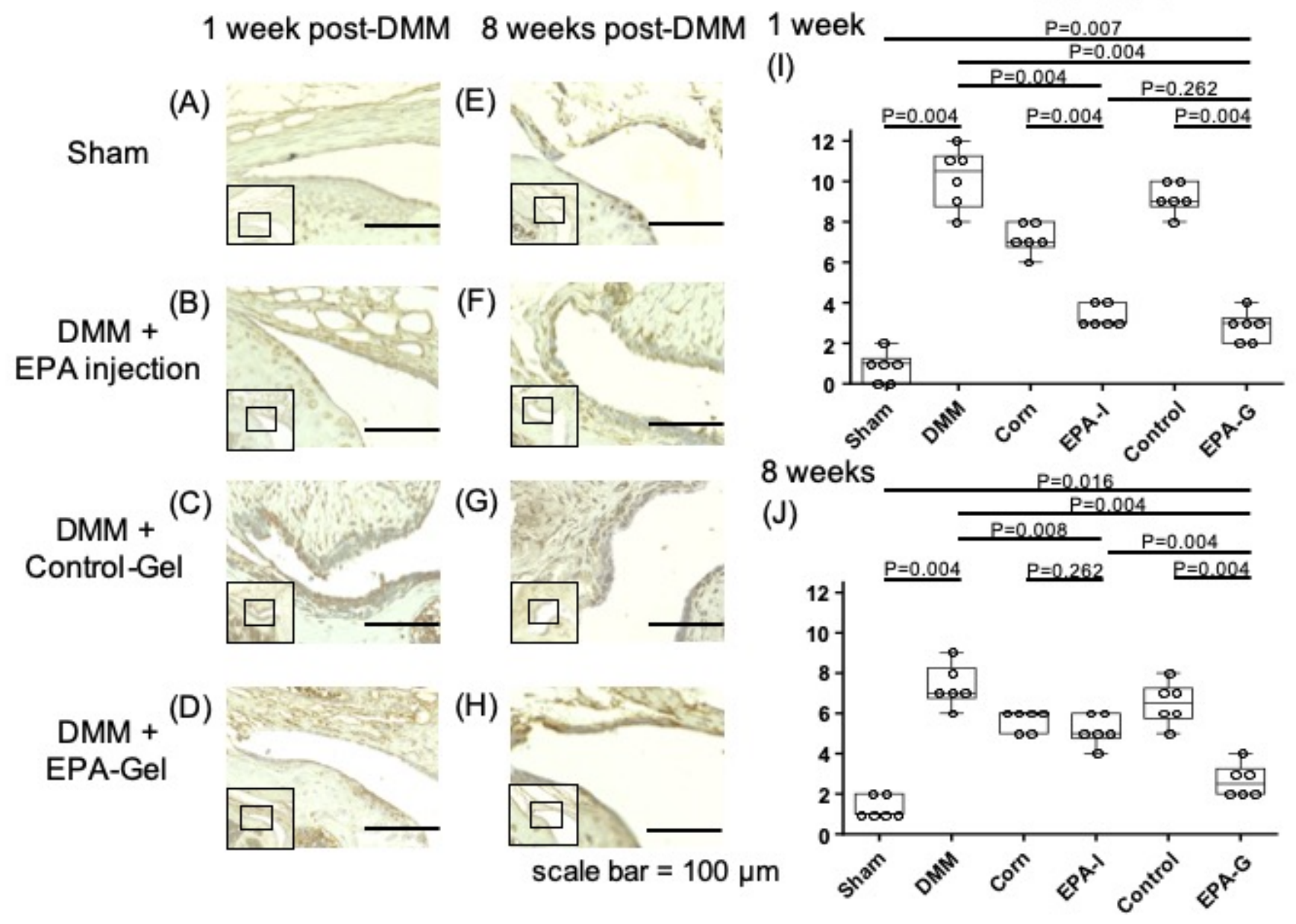

Figure 4

Immunohistochemical analysis using F4/80 score of synovitis EPA was disposed to prevent macrophage infiltration in synovial tissues. Immunohistochemical analysis showed the average-sum F4/80 score of synovitis at 1 week and 8 weeks after surgery. The average sum scores at 1 week showed significant difference between the sham and DMM groups, Corn and EPA-I groups, and Control and EPA-G groups, and no significant difference between the EPA-I and EPA-G groups. The average sum scores at 8 weeks showed significant difference between the sham and DMM groups, Control and EPA-G groups, and EPA-I and EPA-G groups, and no significant difference between the Corn and EPA-I groups.

Immunohistochemical evaluation of F4/80 revealed a significant decrease in OA after EPA treatment, especially in the group treated with EPA-incorporating gelatin hydrogels at 8 weeks after surgery. DMM: destabilization of the medial meniscus, OA: osteoarthritis, EPA: eicosapentaenoic acid 


\section{Figure. 5 Immunohistochemical analysis of CD86}

1 week post-DMM 8 weeks post-DMM

(A)

Sham

$\mathrm{DMM}+$ EPA injection

$\mathrm{DMM}+$

(C)

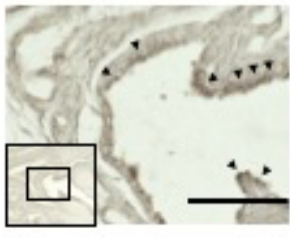

$\mathrm{DMM}+$

(D)

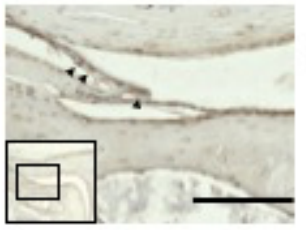

(E)

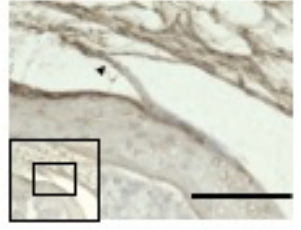

(F)

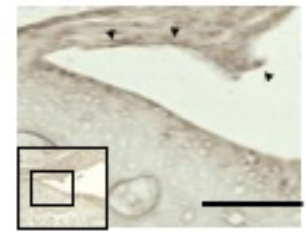

(G)

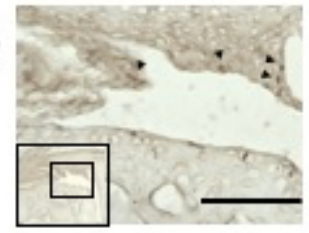

(H)

scale bar $=100 \mu \mathrm{m}$

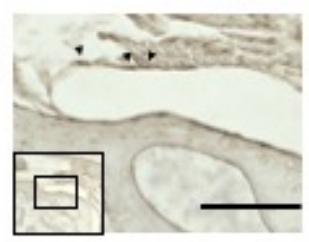

Positive Cell Ratio

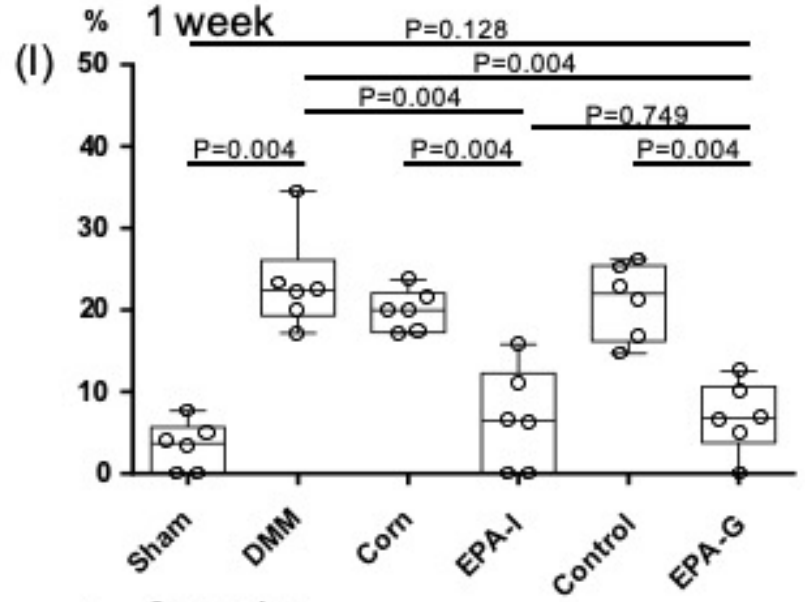

(J)
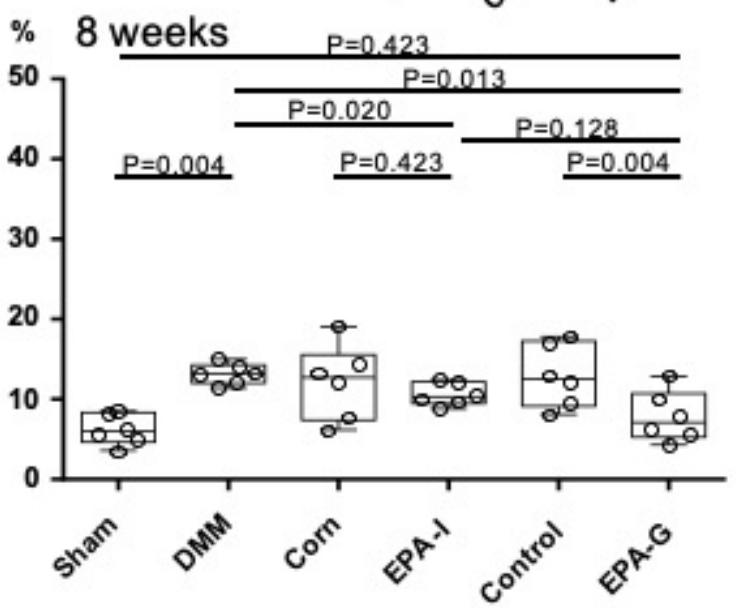

Figure 5

Immunohistochemical analysis using the average-sum ratio of CD86-positive cells Immunohistochemical analysis showed the average-sum ratio of CD86-positive cells at 1 week and 8 weeks after surgery. The ratio of CD86-positive cells at 1 week in the sham, DMM, Corn, EPA-I, Control, and EPA-G groups were 3.3 $\pm 2.7,23.3 \pm 5.4,19.9 \pm 2.3,6.6 \pm 5.7,21.1 \pm 4.2$, and $6.8 \pm 3.9$, respectively, with significant difference between the sham and DMM groups, Corn and EPA-I groups, and Control and EPA-G groups, no significant difference between the EPA-I and EPA-G groups. The ratio of CD86-positive cells at 8 weeks in the sham, DMM, Corn, EPA-I, Control, and EPA-G groups were $6.2 \pm 1.7,13.2 \pm 1.2,12.1 \pm 4.2,10.6 \pm 1.3$, $12.9 \pm 3.6$, and $7.8 \pm 2.9$, respectively, with significant difference between the sham and DMM groups, and Control and EPA-G groups, no significant difference between the Corn and EPA-I groups, EPA-I and EPA-G groups, and sham and EPA-G groups. Immunohistochemical evaluation of CD86 revealed a significant decrease in OA after EPA treatment. Results are presented as mean values with standard deviation. DMM: destabilization of the medial meniscus, OA: osteoarthritis, EPA: eicosapentaenoic acid 
Figure. 6 Immunohistochemical analysis of CD163

1 week post-DMM 8 weeks post-DMM

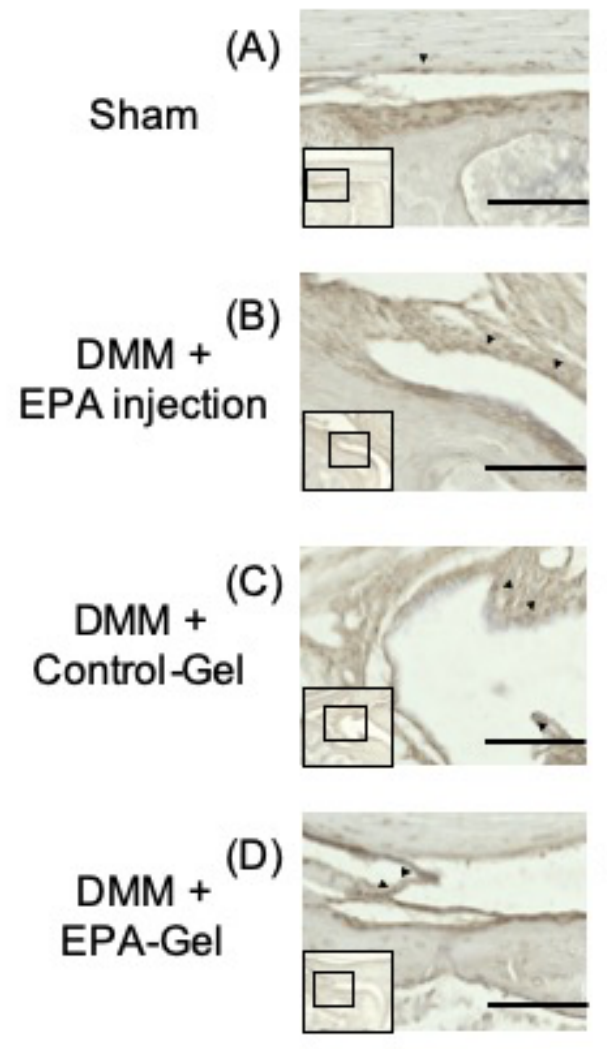

$(\mathrm{E})$

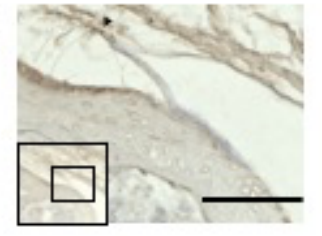

$(\mathrm{F})$

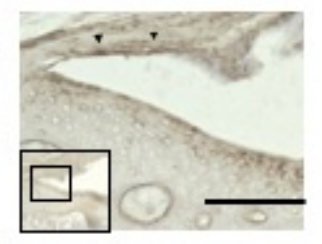

(G)

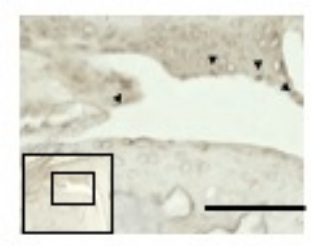

(H)

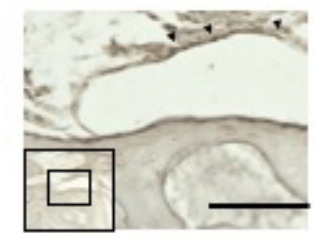

scale bar $=100 \mu \mathrm{m}$
Positive Cell Ratio

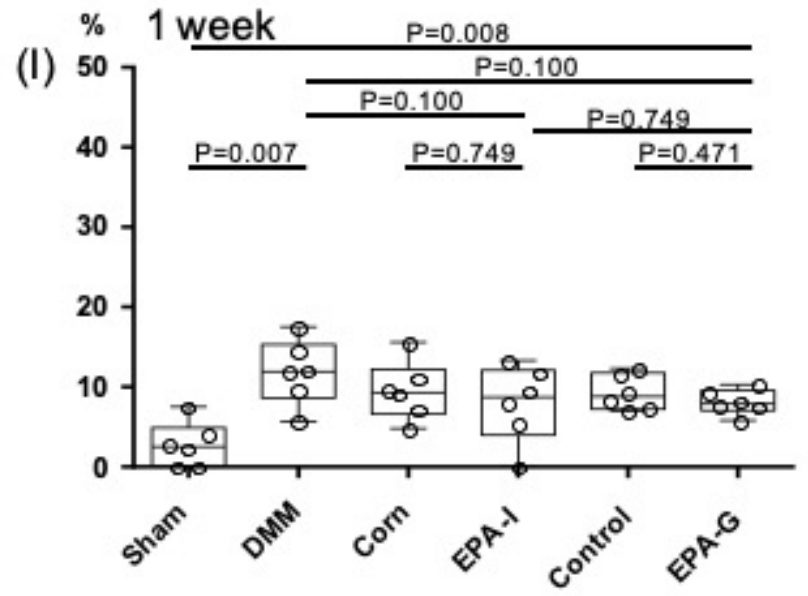

( J)
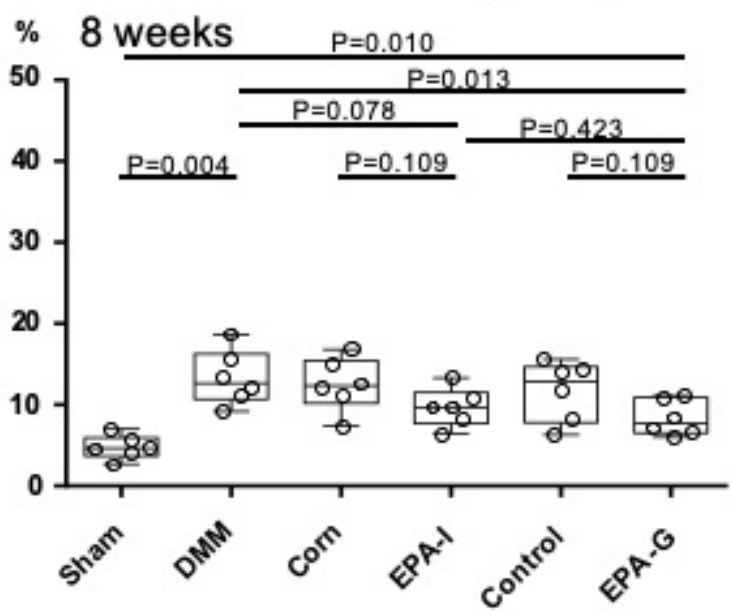

Figure 6

Immunohistochemical analysis using the average-sum ratio of CD163-positive cells Immunohistochemical analysis showed the average-sum score ratio of CD163-positive cells at 1 week and 8 weeks after surgery. The ratio of CD163-positive cells at 1 week in the sham, DMM, Corn, EPA-I, Control, and EPA-G groups were $2.8 \pm 2.6,11.9 \pm 3.7,9.6 \pm 3.3,8.0 \pm 4.4,9.3 \pm 2.0$, and $8.1 \pm 1.4$, respectively, with significant difference between the sham and DMM groups, no significant difference between the Corn and EPA-I groups, Control and EPA-G groups, and EPA-I and EPA-G groups. The ratio of CD86-positive cells at 8 weeks in the sham, DMM, Corn, EPA-I, Control, and EPA-G groups were $4.7 \pm 1.3$, $13.3 \pm 3.1,12.5 \pm 3.0,9.7 \pm 2.2,11.7 \pm 3.4$, and $8.3 \pm 2.0$, respectively, with significant difference between the sham and DMM groups, no significant difference between the Corn and EPA-I groups, Control and EPA-G groups, and EPA-I and EPA-G groups. Immunohistochemical evaluation of CD163 revealed no significant decrease in OA after EPA treatment. Results are presented as mean values with standard deviation. DMM: destabilization of the medial meniscus, OA: osteoarthritis, EPA: eicosapentaenoic acid 
Figure. 7 Immunohistochemical analysis of IL-1 $\beta$, p-IKK, MMP-13 (8 weeks post-DMM)

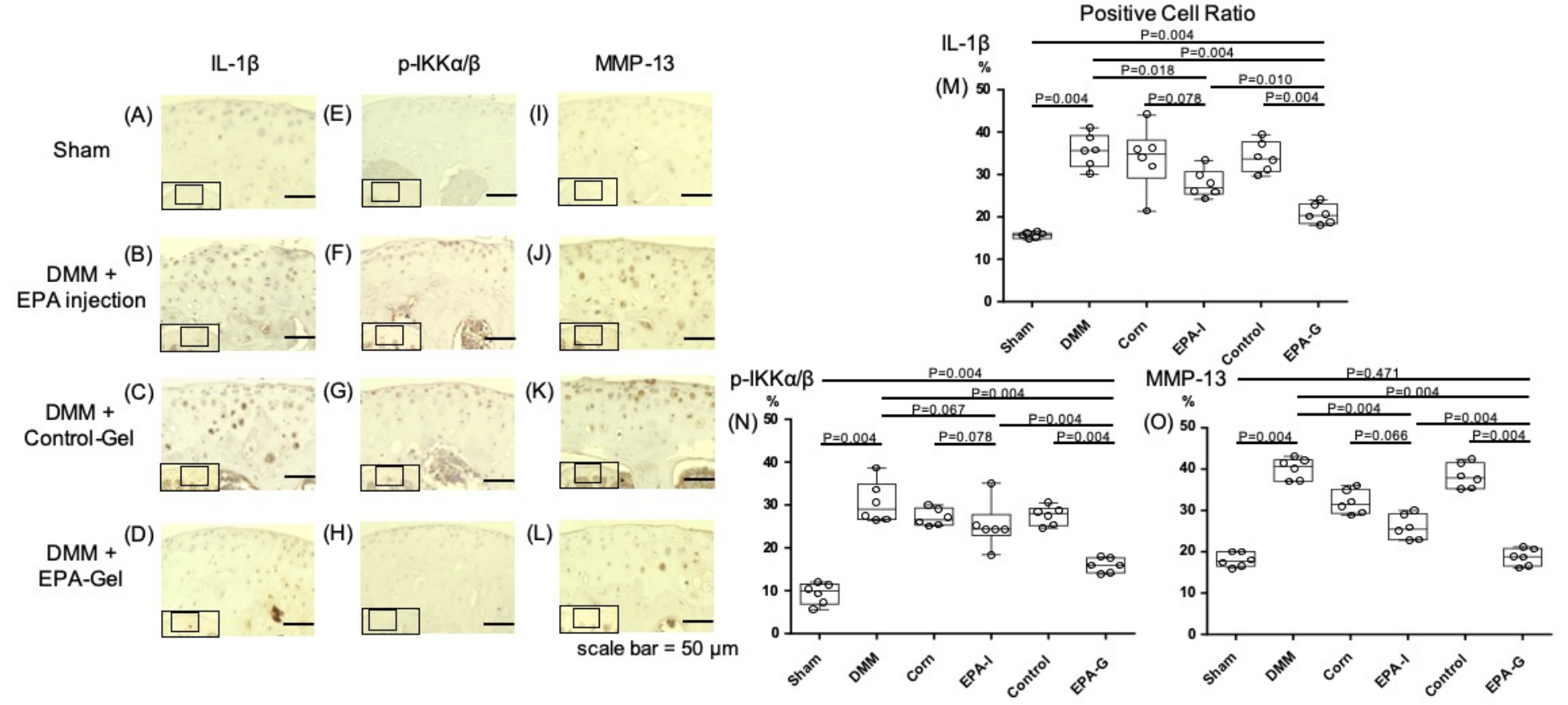

Figure 7

Immunohistochemical analysis using the average-sum ratio of IL-1 $\beta-, \mathrm{p}-\mathrm{IKK} / \mathrm{K} /$-, and MMP-13-positive cells Immunohistochemical analysis showed the average-sum ratio of IL-1 $\beta-$, $p-I K K a / \beta-$, and MMP-13positive cells at 8 weeks after surgery. The ratio of IL-1 $\beta$-positive cells at 8 weeks in the sham, DMM, Corn, EPA-I, Control, and EPA-G groups were $15.6 \pm 0.66,35.6 \pm 3.6,33.8 \pm 6.8,27.8 \pm 3.0,34.1 \pm 3.3$, and $20.7 \pm$ 2.1 , respectively. The ratio of $\mathrm{p}-\mathrm{IKKa} / \beta$-positive cells at 8 weeks in the sham, DMM, Corn, EPA-I, Control, and EPA-G groups were $9.4 \pm 2.3,30.6 \pm 4.4,27.1 \pm 1.8,25.3 \pm 4.9,27.6 \pm 2.0$, and $15.9 \pm 1.5$, respectively. The ratio of MMP-13-positive cells at 8 weeks in the sham, DMM, Corn, EPA-I, Control, and EPA-G groups were $18.0 \pm 1.5,40.1 \pm 2.3,32.0 \pm 2.6,25.9 \pm 2.7,38.3 \pm 2.8$, and $18.7 \pm 1.9$, respectively. The ratios of IL$1 \beta-, \mathrm{p}-\mathrm{IKKa} / \beta-$, and MMP-13-positive cells at 8 weeks showed significant difference in the sham and DMM groups, Control and EPA-G groups, and EPA-I and EPA-G groups, no significant difference in the Corn and EPA-I groups. Immunohistochemical evaluation of IL-1 $\beta, \mathrm{p}-\mathrm{IKK} \mathrm{K} / \beta$ and MMP-13 revealed a significant decrease in OA after EPA treatment. Results are presented as mean values with standard deviation. DMM: destabilization of the medial meniscus, OA: osteoarthritis, EPA: eicosapentaenoic acid 
Figure. 8

(A)

IL1- $\beta \quad 0$ min 5 min $10 \mathrm{~min} 15 \mathrm{~min} 30 \mathrm{~min} 60 \mathrm{~min}$ $\mathrm{p}-\mathrm{IKKa} / \beta$

$87 \mathrm{kDa}$

a-tubulin

$55 \mathrm{kDa}$

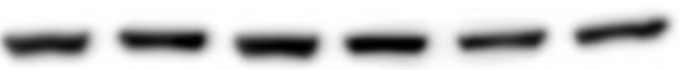

(B) IL1- $\beta \quad 0 \min 15 \mathrm{~min} 15 \mathrm{~min}$

EPA - +

$\mathrm{p}-\mathrm{IKKa} / \beta$

$87 \mathrm{kDa}$

a-tubulin

$55 \mathrm{kDa}$

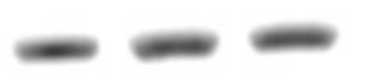

(C) IL1- $\beta \quad 0 \mathrm{~h} 12 \mathrm{~h} 12 \mathrm{~h} 12 \mathrm{~h} 12 \mathrm{~h}$ EPA $\quad$ - + ++ +++

MMP-13

$60 \mathrm{kDa}$

a-tubulin

$55 \mathrm{kDa}$

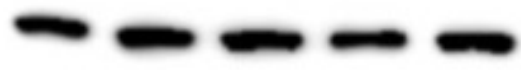

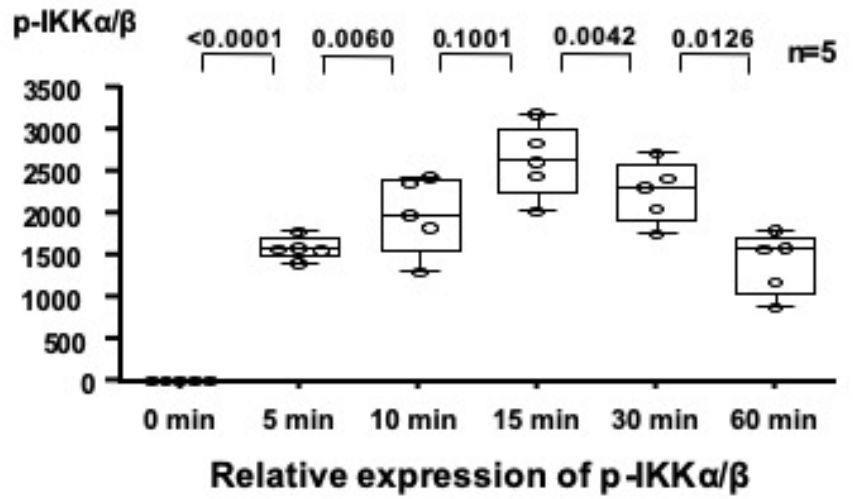

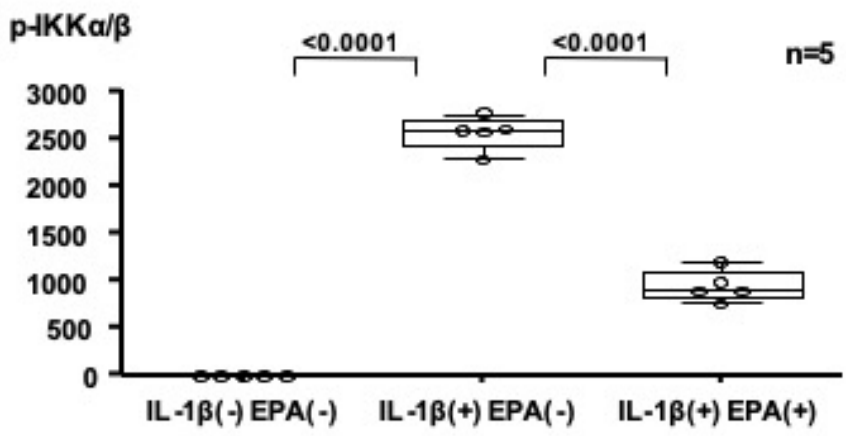

Relative expression of $p-1 K K \alpha / \beta$

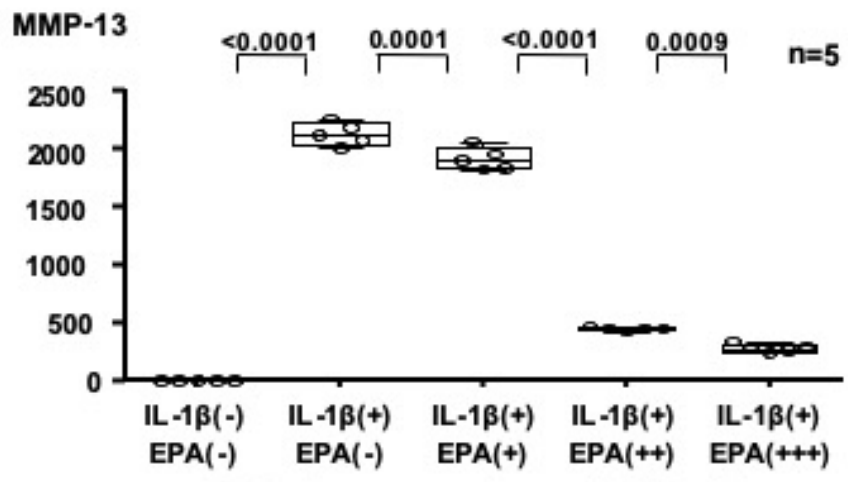

Relative expression of MMP-13

Figure 8

Enhanced expression of inflammatory transcription factors in response to IL $1 \beta$ stimulation in vitro (A) Phosphorylation of $\mathrm{p}-\mathrm{IKKa} / \beta$ in NHAC-kn treated with $10 \mathrm{ng} / \mathrm{ml} \mathrm{IL} 1 \beta$ was assessed. The results confirmed the time dependent change in the phosphorylation of $p-I K K a / \beta$ following IL $1 \beta$ stimulation, and that the phosphorylation levels of $\mathrm{p}-\mathrm{IKKa} / \beta$ were markedly elevated at $15 \mathrm{~min}$ after treatment. $\mathrm{X}$-axis showed that the time of incubation time with IL-1 $\beta$ stimulation. (B) The IL-1 $\beta$-induced expression of $p$ IKKa/ $\beta$ after incubation without EPA was significantly higher compared with that after incubation with EPA. X-axis showed $p-I K K a / \beta$ expressions among three groups; without IL-1 $\beta$ stimulation and EPA incubation, with IL-1 $\beta$ stimulation and without EPA incubation, and with IL-1 $\beta$ stimulation and EPA incubation. (C) The IL-1 $\beta$-induced expression of MMP-13 after incubation without EPA was significantly higher than that after incubation with EPA. Furthermore, the effect was dose-dependent; the higher the dose, the more remarkable the effect. X-axis showed MMP-13 expressions among five groups; without IL- 
$1 \beta$ stimulation and EPA incubation, with IL-1 $\beta$ stimulation and without EPA incubation, with IL-1 $\beta$ stimulation and low-dose EPA incubation, with IL-1 $\beta$ stimulation and medium-dose EPA incubation, and with IL-1 $\beta$ stimulation and high-dose EPA incubation. EPA: eicosapentaenoic acid

\section{Supplementary Files}

This is a list of supplementary files associated with this preprint. Click to download.

- FigureS3.tiff

- Figures2.tiff

- FigureS1.tiff 